\title{
REPORT OF A CASE OF LEPROSY IN A NORFOLK PRACTICE
}

\author{
A. S. Garrett, M.B., B.S., M.R.C.S.(ENG.)
}

A man aged 22 years came to my surgery complaining of a rash on his chest. For some two months he had noticed a plaque 2 inches by 1 inch in size $(2.54 \mathrm{~cm}$. x $5.08 \mathrm{~cm}$.), and many new small raised red lesions had begun to appear a week or two before he came.

The plaque was well-defined, and raised and erythematous, with a slight tendency to central spontaneous healing. The supraclavicular nerve was enlarged leading to the plaque (left internal branch of the nerve). The plaque was anaesthetic. Around the main plaque there were many small raised erythematous lesions with edges less well defined. The left great auricular nerve was enlarged, and the left ear lobe was slightly thicker than the right. Smears were taken from the skin of the ear and of the lesions and showed mild bacterial positivity in 3 out of 5 smears.

Dapsone (DDS) tablets were given at $100 \mathrm{mg}$. twice weekly by mouth, and during the first 3 weeks new lesions continued to appear over the chest and back. Then the inunction of Etisul was begun, and within 2 weeks the lesions had improved markedly, and within a month the erythema and elevation of the lesions had disappeared, and the nerves returned practically to normal size. The anaesthesia over the area of the original lesion persisted until 2 months, and by that time all smears were negative. The only possible sign left is some hypopigmentation of the skin of the back and chest.

\section{Previous history}

The patient had resided 14 months in Cyprus, whence he departed in December 1957. This suggests an incubation period of nearly 3 years from the probable infection.

\section{Summary}

A case of leprosy met with in country practice in England is here briefly described. It seems to be a case of borderline leprosy, in which body resistance was in process of breaking down at the time of presentation for diagnosis of a "rash". In this case it was found there was a very rapid response to therapy by Dapsone (DDS) combined with Etisul by inunction, and this therapy has enabled him to return to work within 2 months.

From my previous experience in Africa, Etisul and DDS combined only acted in this very rapid manner in 2 or 3 very early cases of infectious leprosy. DDS alone would probably have taken about 6 months to achieve this effect. Perhaps bringing in Etisul sooner would have prevented the outcrops of new lesions in this case. 